$$
\text { "ambrus" — 2007/8/17 — 10:37 — page } 231 \text { — \#1 }
$$

\title{
Analyse von Lösungswegen und Erweiterungsmöglichkeiten eines Problems für die Klassen 7-11
}

\author{
Gabriella Ambrus
}

Abstract. Making several solutions for a problem i.e. the generalization, or the extension of a problem is common in the Hungarian mathematics education.

But the analysis of a problem is unusual where the connection between the mathematical content of the task and of its different formulations is examined, solutions from different fields of mathematics are presented regarding the knowledge of different age groups, the problem is generalized in different directions, and several tools (traditional and electronic) for solutions and generalizations are presented.

This kind of problem analysis makes it viable that during the solution/elaboration several kinds of mathematical knowledge and activities are recalled and connected, facilitating their use inside and outside of mathematics.

However, an analysis like this is not unfamiliar to the traditions of the Hungarian problem solving education - because it also aims at elaborating a problem - but from several points of view.

In this study, a geometric task is analysed in such a way.

Key words and phrases: Problemsolving, CABRI, Computer in Teaching of Mathematics, Model, generalization of problems, graphical calculator.

ZDM Subject Classification: D50, E50, G30, U50, U60, U70, N80.

Zu einer Aufgabe mehrere Lösungen bereiten bzw. die Verallgemeinerung oder Weiterdenken der Aufgabe kommt oft im ungarischen Mathematikunterricht vor. Es ist aber nicht üblich eine Aufgabe folgendermaßen zu analisieren:

(1) Untersuchung der Beziehung zwischen der mathematischen Inhalt und mehreren möglichen Texte (Formulierung) der Aufgabe.

(2) Auflisten von Lösungswegen von verschiedenen Gebieten der Mathematik, betrachtend auch die verschiedenen Vorkenntnisse der SchülerInnen in verschiedenen Jahrgängen. 


$$
\text { "ambrus" — 2007/8/17 — 10:37 — page } 232 \text { — \#2 }
$$

(3) Weiterdenken der Aufgabe in verschienen möglichen Richtungen.

(4) Vorstellung und Analyse von verschiedenen Hilfsmitteln (herkömmliche und elektronische) die beim Lösen und Weiterdenken behilflich sein können.

Für die Analyse wurde folgendes Problem gewählt das beim Wettbewerb Tamás Varga 1992 als Aufgabe 4 für SchülerInnen der 7. Klasse gestellt wurde.

Der Mittelpunkt eines Quadrats mit der Seitenlänge 2cm sei ein Eckpunkt eines anderen Quadrats mit der gleichen Seitenlänge.

Wie groß ist der gemeinsame Teil beider Quadrate?

Eine Bearbeitung des Problems für 7. Klasse befindet sich in [2].

\section{Mathematisches Inhalt und Formulierung}

Das ursprüngliche mathematische Inhalt kann verschiedenerweise formuliert werden, das oben angegebene Text (Text 1a) beschränkt sich auf die „Kerninhalt" undzwar in konkreter Kontext (Angabe der Seitenlängen).

In einer anderen Formulierung kann aber statt der konkreten Seitenlängen nur die Verhältnis der Seitenlängen der Quadraten angegeben werden.

Text $1 b$

Es gibt zwei gleichgroße Quadrate. Das eine Quadrat liegt mit einem Eckpunkt auf dem Mittelpunkt des anderen befestigt.

Wie groß ist der gemeinsame Fläche?

Ohne wesentliche Änderung des mathematischen Inhaltes des Problems gibt es weitere Möglichkeiten zur Formulierungen des Textes, zum Beispiel die Folgende:

Text 2

In den Mittelpunkt eines Quadrats mit der Seitenlänge $a$ stecken wir einen Eckpunkt eines anderen Quadrates mit der Seitenlänge $b$. Das zweite Quadrat dreht sich um diesen Punkt. Wie groß ist die überschneidende Fläche?

Text $3 a$

Es gibt zwei gleichgroße Quadrate aus Glas, ein gelbes und ein blaues. Das blaue Quadrat liegt mit einem Eckpunkt auf dem Mittelpunkt des gelben befestigt.

Wie groß ist die grüne Fläche? 


$$
\text { "ambrus" — 2007/8/17 — 10:37 — page } 233 \text { — \#3 }
$$

Text $3 b$

Es gibt zwei gleichgroße quadratförmige Filter. Ein Filter ist mit einem Eckpunkt im Mittelpunkt des anderen befestigt.

Die Filterwirkung ist besser, wenn zwei Filterschichten einander überdecken. In welcher Lage der Quadrate ist die Filterwirkung am größten?

Text 4

Im Mittelpunkt eines Quadrates dreht sich der Eckpunkt eines kongruenten anderen Quadrates.

Wie groß ist die Wahrscheinlichkeit, dass, nachdem das zweite Quadrat herumgewirbelt (und angehalten) wurde, die sich überlappenden Flächenteile der beiden Quadrate ein Viertel des ersten Quadrats ausmacht?

In Text 2 ist das Problem in einer schwereren Formulierung gestellt; wegen der verschiedenen Seitenlängen muss mit Parametern gearbeitet werden, was für die SchülerInnen nicht leicht ist.

Text $1 b$ kann als ein Spezialfall für Text 2 betrachtet werden.

Es handelt sich hier um eine Problemstellung mit „offenem Eingang“. (Zur Problemklassifikation siehe $[6,7,9,10,15,16]$.) Wenn die Aufgabe so formuliert ist, werden die Schüler im Allgemeinen auf „Denken in Zusammenhängen“, oder „Denken mehrseitig“ angeregt.

Schüler, die Aufgaben solcher Art öfter bearbeiten, können die Notwendigkeit der Fallunterscheidung erkennen und können sich daran gewöhnen, in Zusammenhängen und „weitersichtig“ zu denken auch bei konkreten (nicht nur mathematischen) Aufgaben (zum Arbeiten mit „offenen Aufgaben“ vgl. [3]).

Bei den anwendungsnäheren Formulierungen wie Text $3 a$ und Text 3b, können nicht nur mathematische Fachkenntnisse sondern auch Sachkenntnisse gefordert werden.

Text 4 gibt ein konkretes Beispiel, wie verschiedene mathematische Bereiche, hier Geometrie und Wahrscheinlichkeitsrechnung in einer Aufgabe auftreten können.

Verschiedene Textvarianten können weitere Zusammenhänge andeuten und den Charakter des Mathematischen verdeutlichen, indem die Schüler erkennen, dass sowohl unterschiedliche innermathematische Formulierungen als auch außermathematische Problemstellungen (wie hier Glasfarbe, Filterwirkung ... ) mit derselben mathematischen Methode behandelt werden können. Es ist wichtig zu bemerken, dass das Bearbeiten und die Übungsfunktion einer Aufgabe kann auch davon abhängen, wie diese formuliert ist. 


$$
\text { "ambrus" — 2007/8/17 — 10:37 — page } 234 \text { — \#4 }
$$

Gleiche oder ähnliche mathematische Inhalte in verschiedenen Formulierungen können das Bild über Mathematik bei den Schülern positiv beeinflussen, und können Zusammenhänge zwischen den Bereichen der Mathematik und Beziehung zwischen Mathematik und Realität geschaffen werden.

Wenn Texte von den SchülerInnen selbst formuliert werden, kann dies auch motivierend wirken. Meinen Erfahrungen nach, geben die SchülerInnen gerne neue Texte an, auch wenn sie sich für Mathematik wenig interessieren. Die verschiedenen Situationen, die in diesen Texten vorkommen, verhelfen auch die aktive, eventuell realitätsnahe Verwendung von mathematischen Kenntnissen; ein in den Lehrplänen erwünschtes aber nur mangelhaft erreichtes Ziel (vgl. TIMSS, PISA, $[8,13])$.

\section{Verschiedene Lösungswege nach verschiedenen Jahrgängen}

Die folgende Lösungswege brauchen mehr oder weniger mathematische Vorkenntnisse und Grundfähigkeiten. Die angegebenen Stufen sind die Jahrgänge, wo der betroffene Lösungsweg erst erwartet werden kann.

\section{Lösung 1}

Dieser Lösungsweg verwendet die vierfache Drehsymmetrie des Quadrates.

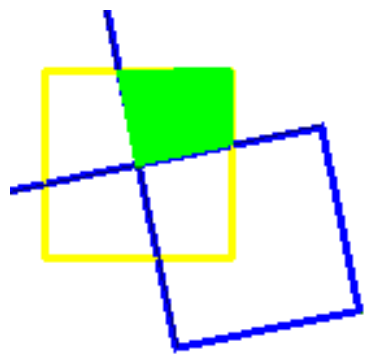

Wenn der gemeinsame Teil um $90^{\circ}, 180^{\circ}, 270^{\circ}$ bzw. $360^{\circ}$ um $O$ gedreht wird, so ist das erste Quadrat in vier Teile gleichmäßig geteilt. Der überlappende Teil ist immer 1/4 des ersten Quadrates.

Die Idee der Verlängerung der Seiten des zweiten Quadrates kann auch aus den Vorkenntnissen z.B. beim Bruchrechnen stammen. 


$$
\text { "ambrus" — 2007/8/17 — 10:37 — page 235 — \#5 }
$$

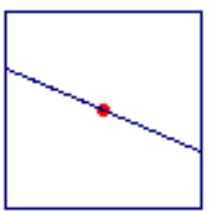

Das Quadrat ist die Einheit. So wird das Quadrat halbiert.

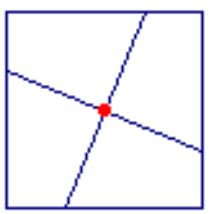

Wenn das Quadrat gleichmäßig noch mal halbiert wird, so ist es in vier gleiche Teile geteilt.

In diesem Alter ist auch zu zeigen, dass der gemeinsame Teil nicht größer als $1 / 4$ sein kann.

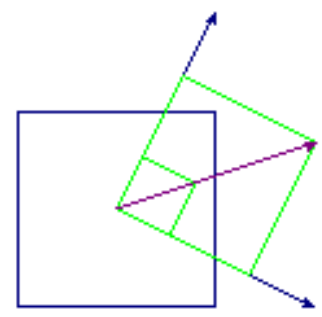

In einer Viertelebene liegt ein Viertel des ersten Quadrates. Das drehende Quadrat liegt innerhalb dieser Viertelebene, so folgt, dass 1/4 eine obere Abschätzung für den überlappenden Teil bei jeder Seitengröße des zweiten Quadrats ist. Ein Maximum soll daher nur in dem Fall untersucht werden, wo der überlappende Teil nie $1 / 4$ erreicht.

Vorkenntnisse: Die vierfache Drehsymmetrie des Quadrates, Zerlegungen in kongruente Teile.

Stufe: 10-12 Jahre (6., 7. Jahrgang) 


$$
\text { "ambrus" — 2007/8/17 — 10:37 — page 236 — \#6 }
$$

Lösung 2

Zum Bestimmen von Flächeninhalten ist die Zerlegung in Dreiecken bzw. in bekannten Flächen schon früher bekannt und verwendet. Das Erkennen von Zusammenhängen, das Aufschreiben und Umformen von Termen sind aber gar nicht leicht, und sind in früheren Jahrgängen nicht zu erwarten.

Der gemeinsame Teil ist in zwei rechtwinklige Dreiecke zerlegt.

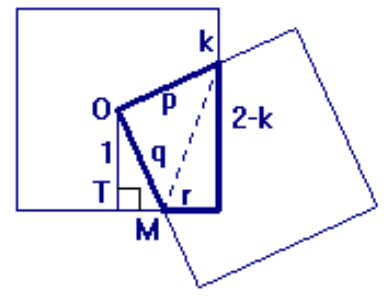

$k=r$ und $p=q$ wegen der Drehsymmetrie des ersten Quadrates um 90 Grad. Aus dem rechtwinkligen Dreieck $O M T$ folgt nach dem Satz des Pythagoras: $p=\sqrt{1^{2}+(1-k)^{2}}$.

Die Fläche des gemeinsamen Teils:

$$
\begin{aligned}
F & =\frac{(2-k) \cdot k}{2}+\frac{p \cdot q}{2} \\
& =\frac{(2-k) \cdot k}{2}+\frac{\left(\sqrt{(1)^{2}+(1-k)^{2}}\right)^{2}}{2}=\frac{2 k-k^{2}+2 \cdot 1-2 k+k^{2}}{2}=1 .
\end{aligned}
$$

Vorkenntnisse: Kongruenz von Dreiecken, Erfahrungen mit Zerlegungen, Fläche von Dreiecken (Drehsymmetrie des Quadrates), Pythagoras, algebraisches Umformen.

Stufe: 14-15 Jahre (8., 9. Jahrgang)

Das Berechnen des gemeinsamen Teils kann auch mit anderen Kenntnissen geschehen.

In der Mittelschule können solche Lösungswege erst erwartet werden wobei mit Koordinaten gerechnet wird. Der Mittelpunkt des Quadrates kommt in den Ursprung des Koordinatensystems. Der gemeinsame Teil wird wie vorher anhand von Teildreiecken bestimmt.

Bei der folgenden zwei Lösungswegen wird die berechende Fläche $(F)$ mit Hilfe von Summe bzw. Differenz von zwei Teildreiecke bestimmt: 
Lösung 3

a)

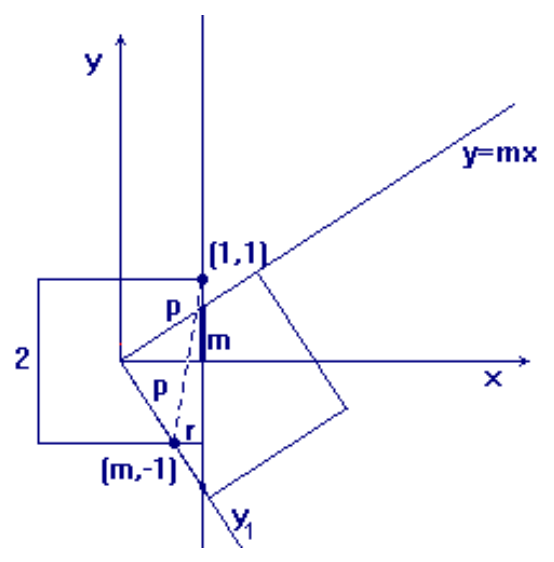

$$
\begin{aligned}
y_{1} & =-\frac{1}{m} x \\
q & =1+m \\
r & =1-m \\
F & =\frac{p^{2}}{2}+\frac{q r}{2} \\
p^{2} & =1+m^{2} \\
F & =\frac{1+m^{2}+1-m^{2}}{2}=1
\end{aligned}
$$

b)

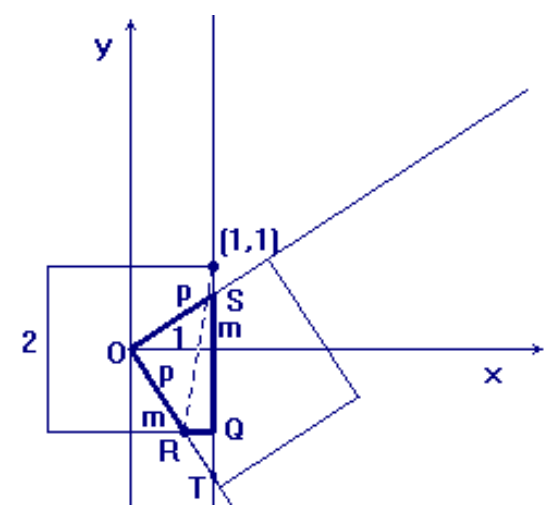

$$
\begin{aligned}
F_{O S T} & =\frac{m+\frac{1}{m}}{2}=\frac{m^{2}+1}{2 m} \\
F_{R Q T} & =\frac{(1-m)\left(\frac{1}{m}-1\right)}{2} \\
& =\frac{(1-m)^{2}}{2 m} \\
& =\frac{1-2 m+m^{2}}{2 m} \\
F & =F_{O S T}-F_{R Q T}=1
\end{aligned}
$$

Vorkenntnisse: Koordinatensystem, die Gleichung der Geraden durch den Ursprung, Zusammenhang von senkrecht stehenden Geraden, Schnittpunkt von Geraden, Flächeninhalt von rechtwinkligen Dreiecken (Hypothenuse · Höhe/2, Kathete $\cdot$ Kathete/2), algebraisches Umformen.

Stufe: 16-17 Jahre (10., 11. Jahrgang)

\section{Weiterdenken, Verallgemeinerung}

Wenn die Seitenlänge des zweiten Quadrates größer als $2 \mathrm{~cm}$ ist, so bleibt der gemeinsame Teil $1 \mathrm{~cm}^{2}$. 


$$
\text { "ambrus" — 2007/8/17 — 10:37 — page 238 — \#8 }
$$

Wenn die Seitenlänge des zweiten Quadrates kleiner als $2 \mathrm{~cm}$ ist, können folgende verschiedene Fälle auftreten:
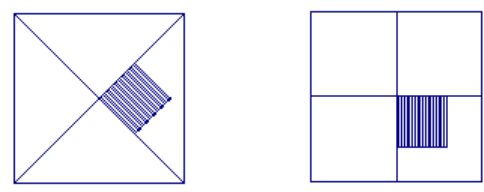

1. Das zweite Quadrat bleibt beim Drehen immer im ersten Quadrat; in diesem Fall ist der gemeinsame Teil die Fläche des zweiten Quadrates.
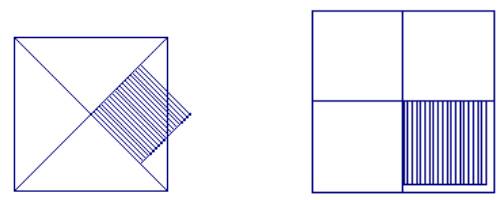

2. Ein Teil des zweiten Quadrates ist beim Drehen manchmal außerhalb des ersten Quadrates, in diesem Fall hängt die Größe des gemeinsamen Teils von der Lage der Quadrate ab.
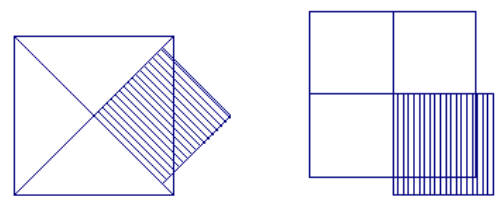

3. Ein Teil des zweiten Quadrates ist beim Drehen immer außerhalb des ersten Quadrates, die Größe des gemeinsamen Teils ist von der Lage der Quadrate abhängig.
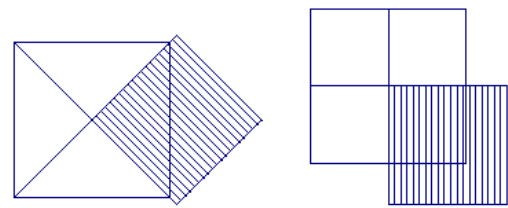

4. Ein Teil des zweiten Quadrates ist immer außerhalb des ersten Quadrates, die Größe des gemeinsamen Teils ist aber immer ein Viertel des ersten Quadrates.

Diese Ergebnisse können in weiteren Jahrgänge präzisiert und ergänzt werden. 


$$
\text { "ambrus" — 2007/8/17 — 10:37 — page 239 — \#9 }
$$

3.1. Arbeiten an der Aufgabe mit Text 2 („größte überlappende Teil“)

Es werden die Parameter $a$ und $b$ für die Seitenlänge des ersten bzw. des zweiten Quadrates entsprechend dem Text 2 der Aufgabe eingeführt.

Bei parametrischen Problemen ist es wichtig, die „wesentlich unterschiedlichen Fälle" herauszufinden. In unserem Beispiel kann man feststellen, dass jede Situation erreicht werden kann, wenn die Seitenlänge des ersten Quadrates konstant gehalten wird und die Seitenlänge des zweiten Quadrates von Null bis Unendlich wächst.

Die folgenden Abschätzungen können für die gemeinsame Fläche unabhängig von der Seitenlänge und der Lage festgestellt werden:

Der Flächeninhalt des gemeinsamen Teiles ist nicht größer als

$a^{2}$ (das stehende Quadrat);

$b^{2}$ (das gedrehte Quadrat);

$\frac{a^{2}}{4}$ (der rechte Winkel enthält einerseits ein Viertel des stehenden Quadrates, andererseits den gemeinsamen Teil).

Je nachdem, ob $b^{2}<\frac{a^{2}}{4}\left(\Leftrightarrow b<\frac{a}{2}\right)$ oder $\frac{a^{2}}{4} \leq b^{2}\left(\Leftrightarrow \frac{a}{2} \leq b\right)$ gilt, erhalten wir als genauere Abschätzung für den überlappenden Teil die Fläche des drehenden Quadrates bzw. das Viertel des stehenden Quadrates.

Falls die Abschätzung der gemeinsamen Fläche durch das zweite Quadrat möglich ist, werden zwei Fälle unterschieden, je nachdem, ob das stehende Quadrat das gedrehte Quadrat immer oder nur in bestimmten Positionen enthält.

- Das zweite Quadrat bleibt beim Drehen immer im ersten Quadrat (spezielle Lage 1a) und in diesem Fall ist der gemeinsame Teil die Fläche des zweiten Quadrates (spezielle Lage 1b).

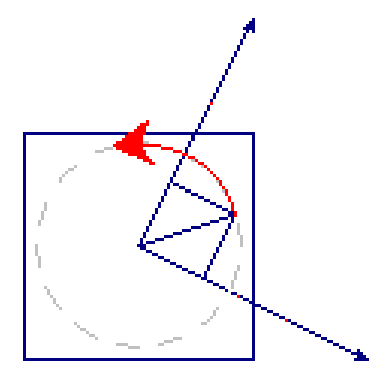
spezielle Lage 1a spezielle Lage $1 \mathrm{~b}$

a

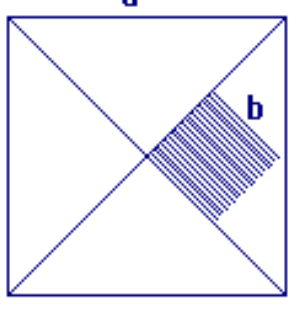

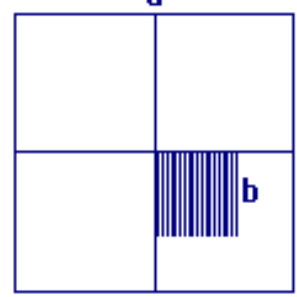


Wenn das zweite Quadrat gedreht wird, bleibt es solange in dem ersten Quadrat, bis seine Diagonale kleiner als die Hälfte der Seitenlänge des ersten Quadrates ist:

$$
\begin{gathered}
b \sqrt{2} \leq \frac{a}{2} \Leftrightarrow \quad b \leq \frac{a}{2 \sqrt{2}} \quad(\text { spezielle Lage 1a) } \\
0<b \leq \frac{a}{2 \sqrt{2}}
\end{gathered}
$$

- Das zweite Quadrat bleibt beim Drehen nur in bestimmten Positionen im ersten Quadrat (spezielle Lagen 2a und 2b). Die Größe der gemeinsamen Fläche ist von der Lage der Quadrate abhängig und kann den maximalen Wert $b^{2}$ erreichen (spezielle Lage 2b).

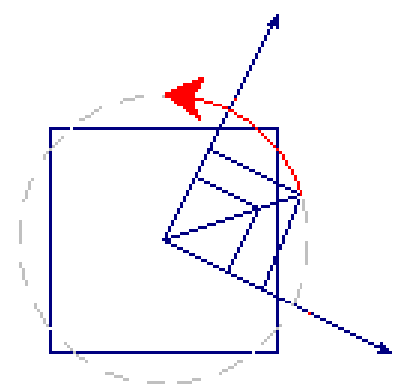

$$
\text { spezielle Lage } 2 \mathrm{a}
$$

spezielle Lage $2 \mathrm{~b}$
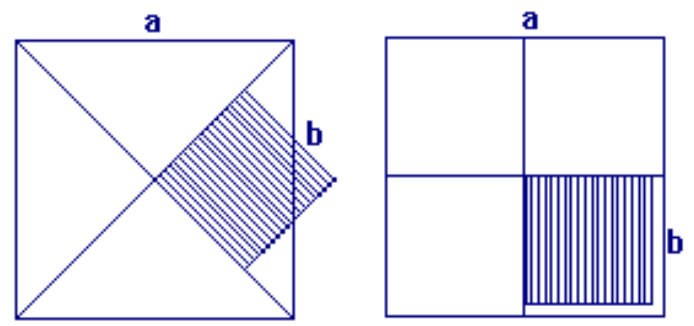

Der Endpunkt der Diagonale des zweiten Quadrates ist beim Drehen manchmal außerhalb des ersten Quadrates, die Diagonale ist größer als die Hälfte der Seite des ersten Quadrates $\left(\frac{a}{2}<b \sqrt{2}\right.$, spezielle Lage 2a).

Die anderen Eckpunkte bleiben immer innerhalb des ersten Quadrates, die Seitenlänge $b$ ist kleiner als $\frac{a}{2}$ (spezielle Lage $2 \mathrm{~b}$ ).

$$
\frac{a}{2 \sqrt{2}}<b<\frac{a}{2}
$$

Falls die Abschätzung der gemeinsamen Fläche durch ein Viertel des ersten Quadrates möglich ist, werden wieder zwei Fälle unterschieden, je nachdem ob das zweite Quadrat während der Drehung ein Viertel des ersten Quadrates nur in bestimmten Positionen oder immer beinhaltet.

- Das zweite Quadrat enthält nur in bestimmten Positionen ein Viertel des ersten Quadrates (spezielle Lage $3 a$ und 3b). Die Größe der gemeinsamen Fläche ist von der Lage der Quadrate abhängig und kann den maximalen Wert $\frac{a^{2}}{4}$ erreichen (spezielle Lage 3b). 


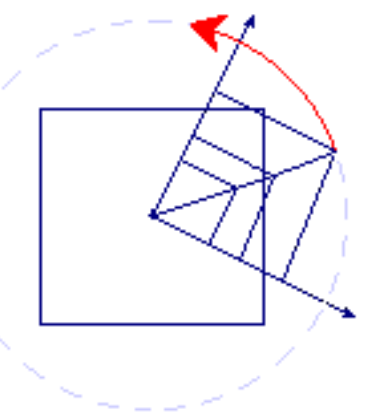

spezielle Lage $3 \mathrm{a}$

spezielle Lage $3 b$
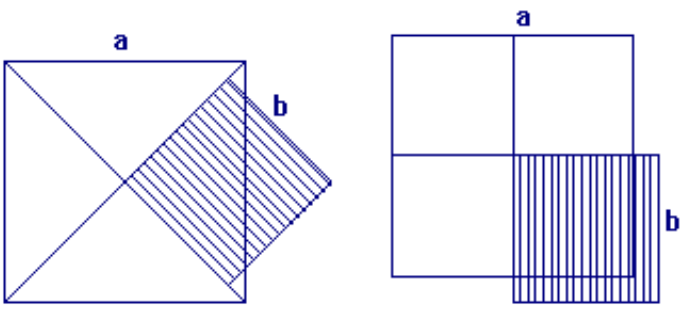

Der Endpunkt der Diagonale des zweiten Quadrates liegt immer außerhalb des ersten Quadrates, zwei anderen Eckpunkte bleiben nur in gewissen Positionen innerhalb des ersten Quadrates $\left(\frac{a}{2} \leq b<\frac{a \sqrt{2}}{2}\right.$, spezielle Lage 3a und 3b).

$$
\frac{a}{2} \leq b<\frac{a \sqrt{2}}{2}
$$

- Ein Viertel des ersten Quadrates ist immer im zweiten Quadrat beinhaltet. Die Größe des gemeinsamen Teils ist immer ein Viertel des ersten Quadrates.

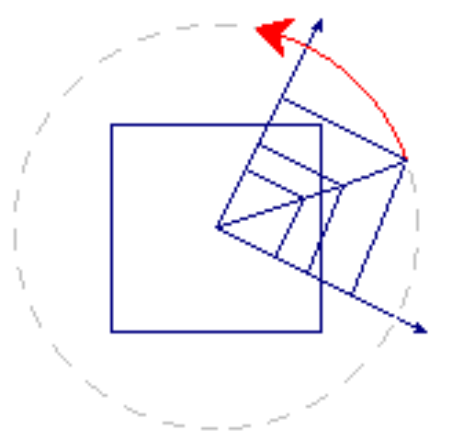

spezielle Lage $4 \mathrm{a}$ spezielle Lage $4 \mathrm{~b}$
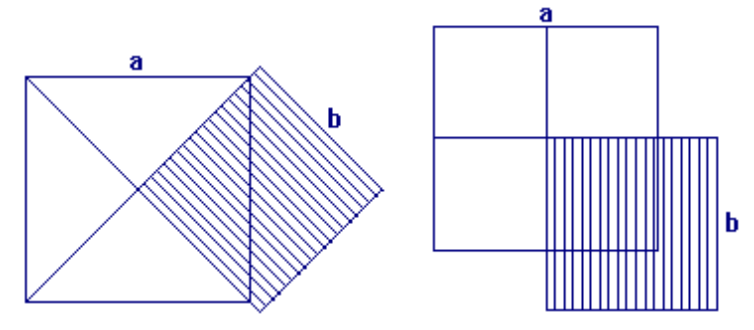

Wenn die Seitenlänge des zweiten Quadrates größer ist als die Hälfte der Diagonale des ersten Quadrates (spezielle Lage 4a), liegt das in dem rechten Winkel enthaltene Viertel des ersten Quadrates innerhalb des zweiten Quadrates.

$$
\frac{a \sqrt{2}}{2} \leq b
$$


Damit wurden die unendlich vielen Fälle in vier Klassen eingeordnet:

\begin{tabular}{|l|l|}
\hline $0<b \leq \frac{a}{2 \sqrt{2}}$ & $\begin{array}{l}\text { Der gemeinsame Teil ist konstant während der Drehung und } \\
\text { gleich mit dem zweiten Quadrat. }\end{array}$ \\
\hline$\frac{a}{2 \sqrt{2}}<b<\frac{a}{2}$ & $\begin{array}{l}\text { Der gemeinsame Teil ändert sich während der Drehung bis } \\
\text { zum zweiten Quadrat. }\end{array}$ \\
\hline$\frac{a}{2} \leq b<\frac{a \sqrt{2}}{2}$ & $\begin{array}{l}\text { Der gemeinsame Teil ändert sich während der Drehung bis } \\
\text { zum Viertel des ersten Quadrates. }\end{array}$ \\
\hline$\frac{a \sqrt{2}}{2} \leq b$ & $\begin{array}{l}\text { Der gemeinsame Teil ist konstant während der Drehung und } \\
\text { gleich mit dem Viertel des ersten Quadrates. }\end{array}$ \\
\hline
\end{tabular}

Da für jeden Parameterwert der größte überlappende Teil angegeben ist, ergibt sich die Frage nach dem kleinsten überlappenden Teil bei $\frac{a}{2 \sqrt{2}}<b<\frac{a}{2}$ bzw. bei $\frac{a}{2} \leq b<\frac{a \sqrt{2}}{2}$.

Im folgenden wird die Größe der überlappenden Fläche als Funktion der Position für $\frac{a}{2 \sqrt{2}}<b<\frac{a}{2}$ untersucht. Durch verschiedene Lösungswege wird die Lage der beiden Quadrate bestimmt, wo der überlappende Teil minimal ist.

\subsection{Untersuchung mit Zerlegungsgleichheit}

(„Der Flächeninhalt als eine Funktion des Drehwinkels“)

Als Variable wird der Schnittwinkel $\alpha$ der Diagonale des stehenden Quadrates $O B$ mit der Seite des gedrehten Quadrates $R Q$ gewählt.

Solange die Diagonale $O Q$ die Seite AB nicht schneidet, bleibt der Flächeninhalt des gemeinsamen Teiles $b^{2}$.

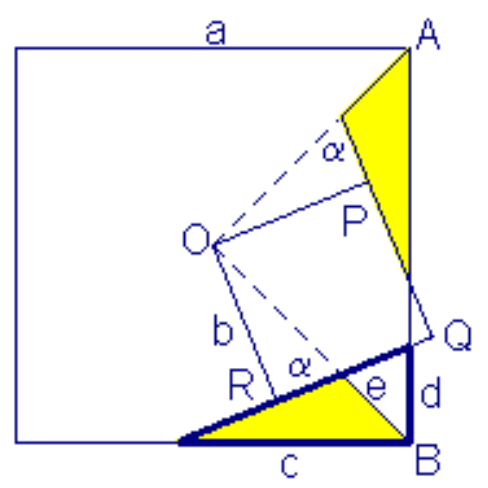


$Q$ liegt außerhalb des stehenden Quadrates genau dann, wenn

$$
\sin \alpha \cdot b \sqrt{2}>\frac{a}{2}
$$

gilt.

Folglich untersuchen wir den Flächeninhalt für

$$
\alpha \in\left[\alpha_{0}=\arcsin \frac{a}{2 \sqrt{2} b} ; 90^{\circ}\right] .
$$

Um den Flächeninhalt des gemeinsamen Teils zu bestimmen, muss (wegen der Zerlegungsgleichheit) aus dem Viertel des ersten Quadrates das mit dicker Linie markierte Dreieck weggelassen werden.

Die Seite $R Q$ schneidet eine Strecke $e$ der Diagonale $O B$ ab, für die $e=\frac{a \sqrt{2}}{2}-\frac{b}{\sin \alpha}$ gilt.

Für die Katheten des mit dicker Linie markierten Dreiecks gelten nach dem Sinussatz $\frac{d}{e}=\frac{\sin \alpha}{\sin \left(135^{\circ}-\alpha\right)}$ und $\frac{c}{e}=\frac{\sin \alpha}{\sin \left(\alpha-45^{\circ}\right)}$.

Durch äquivalentes Umformen

$$
\begin{gathered}
\sin \left(135^{\circ}-\alpha\right)=\sin 135^{\circ} \cdot \cos \alpha-\sin \alpha \cdot \cos 135^{\circ}=\frac{1}{\sqrt{2}} \cos \alpha+\frac{1}{\sqrt{2}} \sin \alpha, \\
\sin \left(\alpha-45^{\circ}\right)=\sin \alpha \cdot \cos 45^{\circ}-\cos \alpha \cdot \sin 45^{\circ}=\frac{1}{\sqrt{2}}(\sin \alpha-\cos \alpha)
\end{gathered}
$$

und Einsetzen von $e$ erhalten wir $d$ in der Form

$$
d=\frac{a \cdot \sin \alpha-b \sqrt{2}}{\cos \alpha+\sin \alpha} \text {. }
$$

Ähnlicherweise ergibt sich für $c$

$$
c=\frac{a \cdot \sin \alpha-b \sqrt{2}}{\sin \alpha-\cos \alpha}
$$

und daraus der Flächeninhalt des bezeichneten Dreiecks

$$
F_{0}=\frac{c d}{2}=\frac{(a \cdot \sin \alpha-b \sqrt{2})^{2}}{-2 \cos 2 \alpha}=\frac{(a \cdot \sin \alpha-x \sqrt{2})^{2}}{4 \sin ^{2} \alpha-2}=\frac{(\sqrt{2} \sin \alpha-x)^{2}}{2 \sin \alpha-1} .
$$

Nach dieser Formel kann eine Funktion $F_{0}(\alpha)$ über dem Definitionsbereich $\left[\alpha_{0}=\arcsin \frac{a}{2 \sqrt{2} b} ; 90^{\circ}\right]$ erklärt werden: $F(\alpha)=\frac{1}{4} a^{2}-F_{0}$.

Für SchülerInnen, die keine Differenzialrechnung lernen, können die Parameter $a$ und $b$ der vorigen Funktion mit Hilfe von CABRI oder von einem CAS untersucht werden (siehe 4.2, und 4.3).

(In ungarischen Mittelschulen hängt es von dem Schultyp ab, ob Differenzialrechnung zum Lehrstoff gehört.) 


$$
\text { "ambrus" — 2007/8/17 — 10:37 — page } 244 \text { — \#14 }
$$

\section{Hilfsmitteln beim Lösen und Weiterdenken}

Die Lösung des Problems ist gar nicht leicht. Nach dem Bedenken von möglichen Lösungswegen in verschiedenen Jahrgängen der Lehrer kann entscheiden welche Hilfe für die SchülerInnen angeboten wird. Diese Hilfe kann eine Frage, Bestätigung, Lösungssegment sein, oder in Zusammenhang mit den Vorigen kann auch Hilfsmittel angeboten werden.

\subsection{Das herkömmliche Modell}
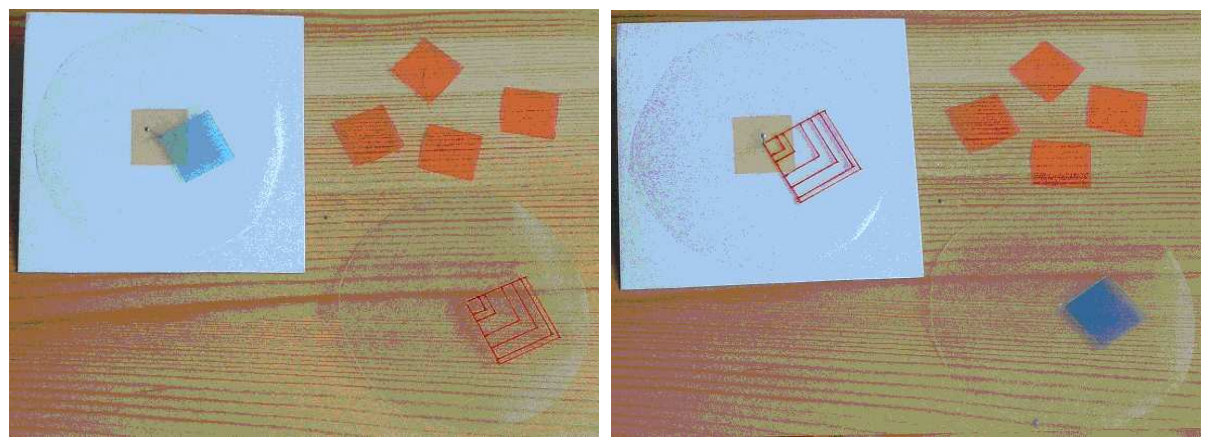

Das gelbe Quadrat ist auf dem weißen großen Kartonquadrat, das blaue Quadrat ist am Folienkreis angeklebt. Der Kreis ist mit Hilfe einer Nadel im Mittelpunkt des gelben Quadrates befestigt. So kann das zweite Quadrat um einen seiner Eckpunkte gedreht werden.

Der Folienkreis kann gewechselt und mit abwaschbarem Filzstift beschrieben werden. Die weiteren kleinen farbigen Quadrate sind bei den drehsymmetrischen Überlegungen behilflich (am Modell können zum Beispiel drei so gelegt werden, dass diese mit dem blauen Quadrat zusammen ein größeres Quadrat bilden).

(Verwendetes Material: Karton, Folien (durchsichtig, gelb, blau und rot), Filzstifte, Stecknadel, Klebestoff.)

Die Untersuchung von Funktionen $F(\alpha)$ bzw. kann geeignete CABRI Figur auch helfen, wobei die Wirkung der Änderung von Parameter $a$ und $b$ gefolgt werden kann. 


\subsection{Untersuchung mit dem CABRI}

Die folgenden Bilder sind Bildschirmausschnitte aus einer CABRI Datei wobei die funktionale Abhängigkeit des Flächeninhaltes $F(\alpha)$ von der Variablen $\alpha$ und die Wirkung der Änderung von den Parametern $a$ und $b$ dynamisch untersucht werden.
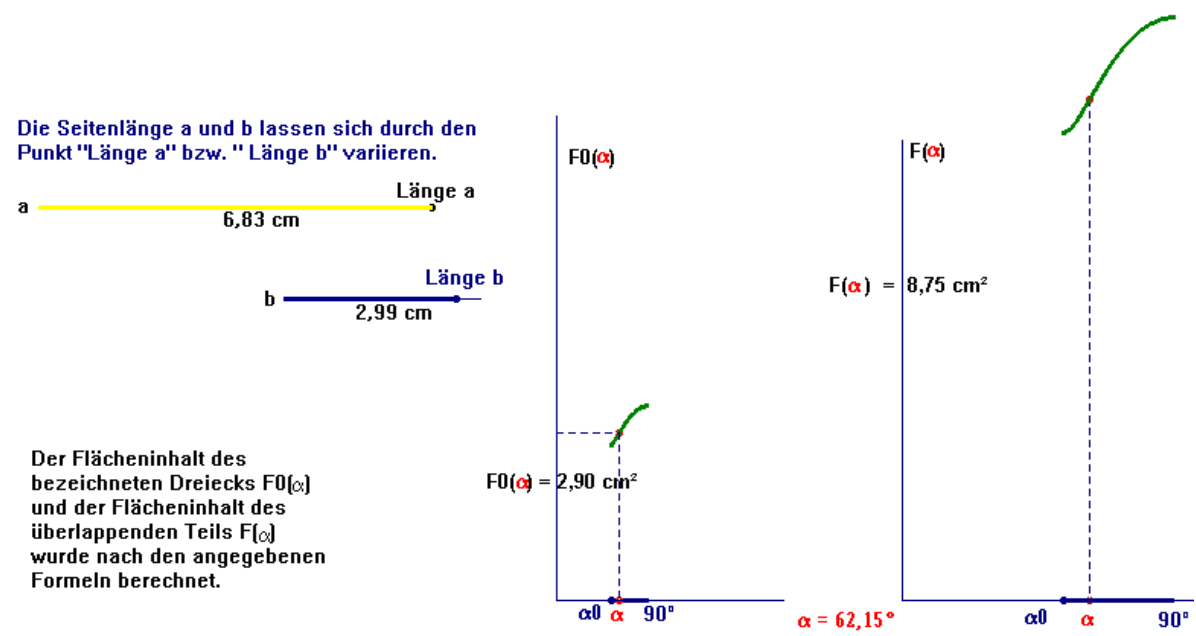

$F(\alpha)$

durch

Bewege $\alpha$

Die Länge von $a$ und $b$ kann mit den schwarzen und blauen Punkten geänder werden. Bei konkreten Werten für $a$ und $b$ kann die Funktion $F_{0}(\alpha)$ im angegebenen Bereich (blaue Strecke auf der $x$-Achse) untersucht werden. 


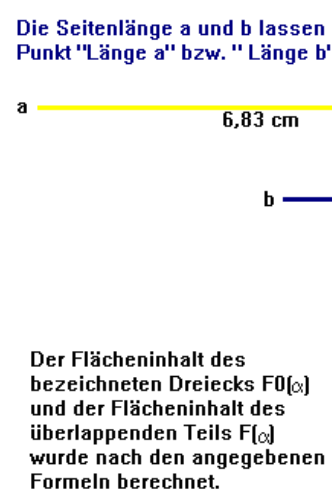

durch den Länge a
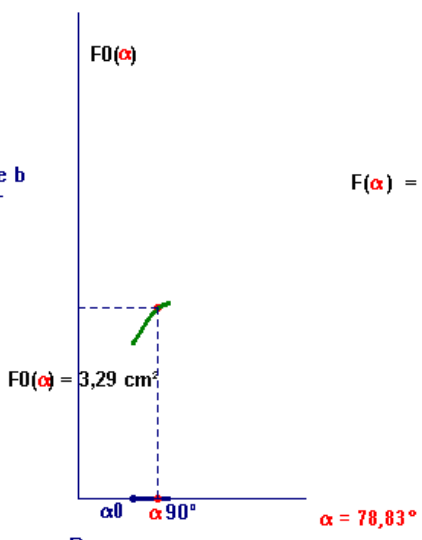

$F(\alpha)$

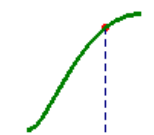

Bewege o

Mit dem Bewegen des Punktes $\alpha$ (an der blauen Strecke auf der $x$-Achse) können die aktuellen Funktionswerte $F_{0}(\alpha)$ und $F(\alpha)$ abgelesen werden.
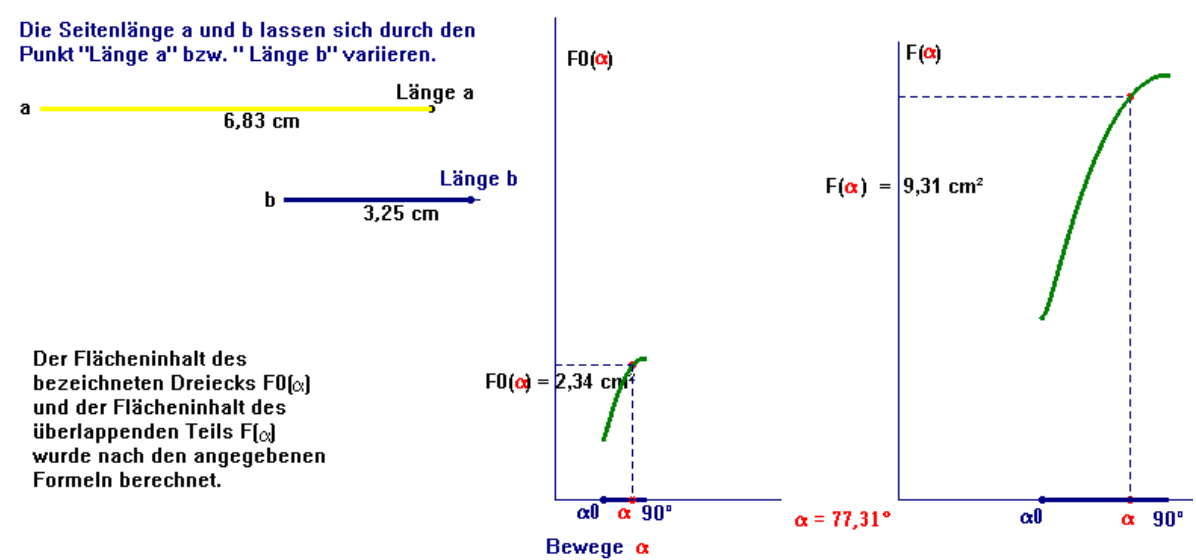

Mit der Änderung der Länge von $b$ in dem von $a$ bestimmten Bereich ändert sich auch $\alpha_{0}$ (der linke Endpunkt) sowie die Länge der blauen Strecke (auf der $x$-Achse) und die Funktionswerte von $F_{0}(\alpha)$ und $F(\alpha)$. 

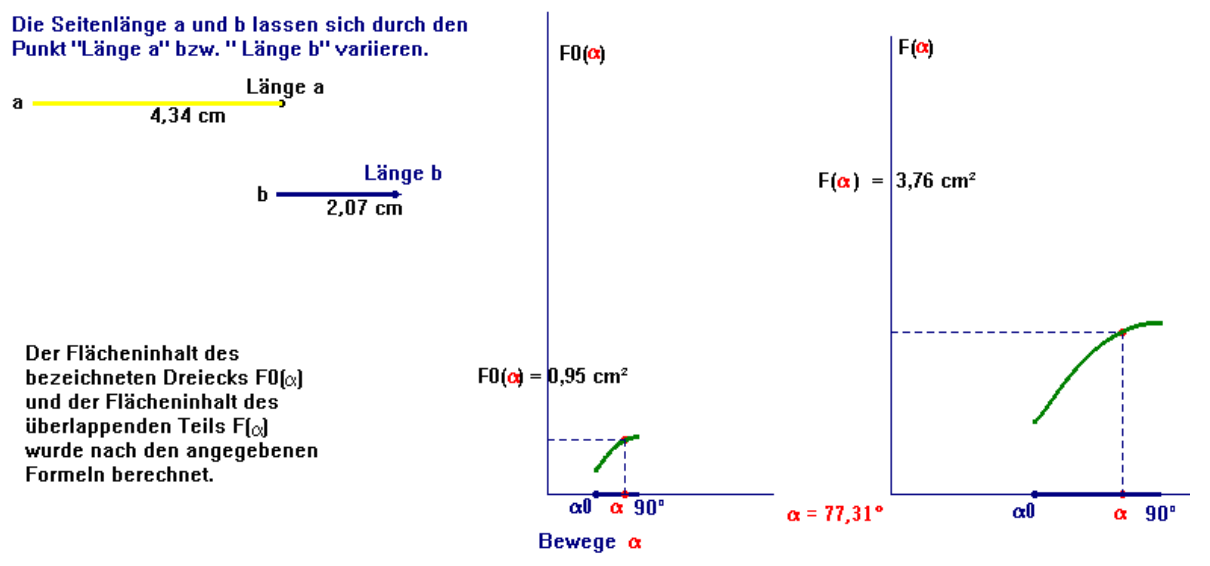

Mit Änderung der Länge von $a$ ändert sich der zu untersuchende Längenbereich von $b$, die „blaue Strecke auf der $x$-Achse“ bleibt unverändert; die Funktionswerte $F_{0}(\alpha)$ und $F(\alpha)$ ändern sich.

Aus der vorigen Untersuchung ergibt sich die Vermutung, dass die Funktion $F_{0}(\alpha)$ bei jedem möglichen Parameterwert ein Maximum hat, dieser wird bei $\alpha=90^{\circ}$ erreicht.

\subsection{Untersuchung mit dem Grafikrechner}

Mit Grafikrechnern können wir nur bei konkreten Werten für $a$ und $b$ die Funktion $F_{0}$ untersuchen. In folgendem haben wir $a=6,83 \mathrm{~cm}$ und $b=2,99$ $\mathrm{cm}$ gewählt, wie bei CABRI. Die Variable $x$ ist bei dieser Einstellung in Grad gemessen. In dem Eingabefenster wird die Formel TI 83 „taschenrechnergerecht" eingetippt. Damit ergibt sich:

$$
F_{0}(x)=\left(\frac{6,83}{2} \cdot \sqrt{2} \cdot \sin (x)-2,99\right)^{2} /\left(2 \cdot[\sin (x)]^{2}-1\right) .
$$

Mit Hilfe der ZOOMBOX kann man Teile der Funktion auch näher untersuchen. Bei $x=45$ gibt es keinen Funktionswert: das vierte (vergrößerte) Bild zeigt an dieser Stelle eine senkrechte Gerade.

Der TRACE Mode erlaubt die Untersuchung des Verlaufs der Funktion. Die Umgebung, wo die Eigenschaften genauer zu untersuchen sind, kann mit dem ZOOM Befehl bis zur Grenze der Aufösung vergrößert werden. Im Kalkulationsmenü kann man die numerische Berechnung des Extremumswertes wählen 

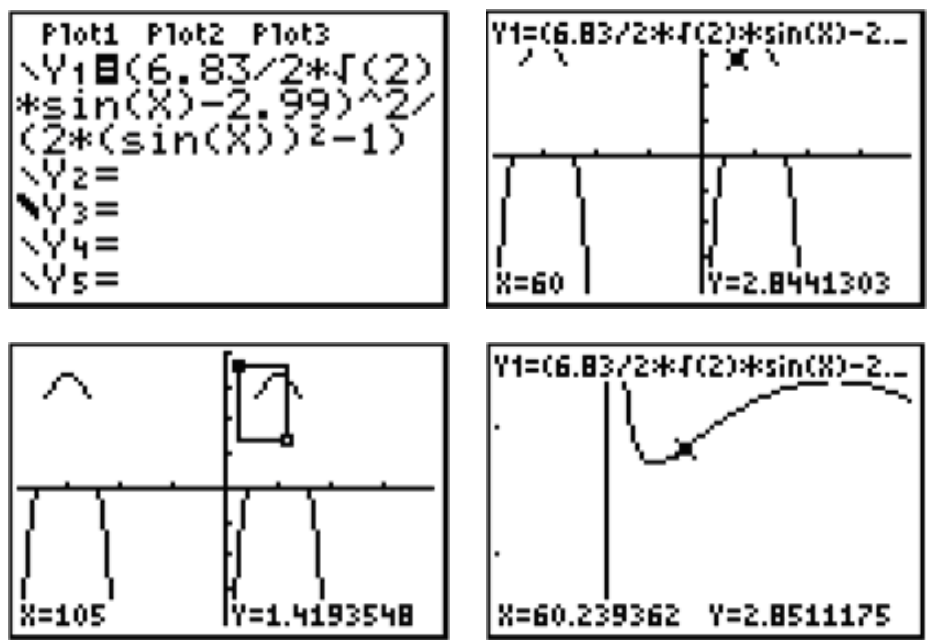

(BLACK-BOX-METHODE). Die Methode der Differentialrechnung kann auch bei der numerischen Untersuchung benutzt werden (GREY-BOX-METHODE).

Es gibt auch andere Methoden, die ohne Differentialrechnung den Extremwert erkennen lassen (siehe: [12]).

Ich bin der Meinung, dass eine Analyse von solcher Art ist gar nicht fremd von den Traditionen des ungarischen Mathematikunterrichtes. Es handelt sich nämlich auch in diesem Fall um einen detaillierten Bearbeiten eines Problems, aber aufgrund noch mehr Gesichtspunkten als es üblich ist. Solche Analyse können erwartungsgemäß die Methode und Mittel von LehrerInnen weiter bereichern.

\section{Literatur}

[1] G. Ambrus, Üben in der Planung des Mathematikunterrichts, Dissertation, Salzburg, 2003.

[2] G. Ambrus, Eine neue Verwendung von elektronischen und herkömmlichen Mitteln am Beispiel der Lösung eines geometrischen Problems in der Klasse 7, in: Positionen-Mathematikdidaktik in Entwicklung, (K. J. Parisot und É. Vásárhelyi, Hrsg.), Abacus, Salzburg, 2005.

[3] G. Ambrus, Nyitott és nyitható feladatok a tanárképzésben és a matematikaoktatásban, [Offene Aufgaben und Öffnung von Aufgaben in der Lehrerausbildung und im Mathematikunterricht], A Matematika Tanítása 8, no. 1 (2000), 7-15. 


$$
\text { "ambrus" — 2007/8/17 — 10:37 — page } 249 \text { — \#19 }
$$

[4] G. Ambrus, CABRI-Geometria, segítőtárs a geometria tanulásában, [CABRI- Geometrie Hilfe zum Lernen Geometrie], A Matematika Tanitása 6, no. 5 (1998), 3-7.

[5] G. Ambrus, Mire használjuk (és mire ne) a CABRI-geometriát, [Wofür verwenden (und wofür nicht die CABRI-Geometrie], Matematikatanár-képzés, Matematikatanár-továbbképzés (November 1999), 55-62.

[6] W. Blum, B. Wiegand, Offene Probleme für den Mathematikunterricht - Kann man Schulbücher dafür nutzen?, Vortrag an 33. GDM Tagung, Bern, 1999.

[7] M. Buth, Lerntheorien (mit Anwendungen im Mathematikunterricht und im Naturwissenschaftlichen Aufgabenfeld), Franzbecker, 1995.

[8] B. Csapó, Az iskolai tudás, [Das schulische Wissen], Osiris Kiadó, Budapest, 1998.

[9] I. Hortobágyi, Aufgabenlösen im problemlösungsorientierten Mathematikunterricht, in: Integrativer Unterricht in Mathematik, (K. J. Parisot und É. Vásárhelyi, Hrsg.), Abakus Verlag, Salzburg, 1997, 115-122.

[10] E. Pehkonen, Introduction: Use of open-ended problems, ZDM 27, no. 2 (April 1995), 55-57.

[11] F. Pogáts, Varga Tamás matematikai versenyek, [das Wettweberb „Varga Tamás"], Typotex, Budapest, 1995.

[12] H. Schumann, É. Vásárhelyi, Computerunterstützte Behandlung geometrischer Extremwertaufgaben, CD-ROM, T⿱3 Hungary, Budapest, 2002.

[13] P. Vári, I. Bánfi, E. Felvégi, J. Krolopp, Cs. Rózsa, T. Szalay, A PISA 2000 vizsgálatról [Über die Untersuchung PISA 2000, ungarisch], Új Pedagógiai Szemle, no. 12 (2001).

[14] É. Vásárhelyi, Paraleller Einsatz von traditionellen Anschauungsmitteln und Computeranimationen als Strategie für Problemlösen, in: Creativity and Mathematics Education, Tagungsband, Münster, 1999, 270-273.

[15] F. Zech, Grundkurs Mathematikdidaktik, Beltz Verlag, 1996.

[16] B. Zimmermann, Problemorientierter Unterricht, Franzbecker, 1991.

GABRIELLA AMBRUS

EÖTVÖS LORAND UNIVERSITÄT

NATURWISSENSCHAFTLICHE FAKULTÄT

MATHEMATISCHES INSTITUT

MATHEMATIKDIDAKTISCHES ZENTRUM

H-1117 BUDAPEST PÁZMÁNY PÉTER SÉTÁNY 1/C

HUNGARY

E-mail: ambrusg@cs.elte.hu

(Received February, 2006) 